\title{
Reflectance-based Classification of Color Edges
}

\author{
Theo Gevers \\ Informatics Institute \\ Faculty of Science \\ Amsterdam, The Netherlands
}

\begin{abstract}
We aim at using color information to classify the physical nature of edges in video. To achieve physics-based edge classification, we first propose a novel approach to color edge detection by automatic noise-adaptive thresholding derived from sensor noise analysis. Then, we present a taxonomy on color edge types. As a result, a parameter-free edge classifier is obtained labeling color transitions into one of the following types: (1) shadow-geometry, (2) highlight edges, (3) material edges. The proposed method is empirically verified on images showing complex real world scenes.
\end{abstract}

\section{Introduction}

The color of objects vary with changes in illuminant color and viewing conditions. As a consequence, color boundaries are generated by a large variety of imaging variables such as shadows, highlights, illumination and material changes. Therefore, classifying the physical nature of edges is useful for a large number of applications such as video segmentation, video indexing and content recognition, where corresponding edge types (e.g. material transitions) from distinct image frames are selected for image matching while discounting other "accidental" edge types (e.g. shadows and highlight transitions). In this paper, we consider the problem of discriminating different edge types based on local surface reflectance properties.

Edge detection in intensity images is well established, [1], [2] for example. In general, to achieve color edge detection, these intensity-based edge detection techniques are extended by taking the sum or Euclidean distance from the individual gradient maps. Further, to obtain robustness against illumination effects, Tsang and Tsang [3] show that edge detection in the hue color space is effective in suppressing specular reflection. However, no edge classification scheme is provided. Another approach is given by Zhang and Bergholm [4] to classify edges into diffuse and sharp edges. The idea is that illumination phenomena, such as indoor shadows and reflections on glossy surfaces tend to cause gradual transitions, whereas edges between distinct objects tend to be sharp. A similar but more elaborated approach is given by Stander [5] for detecting moving shadows. However, the method is based on a complex geometry model restricted to the detection of cast shadows. Furthermore, all of the above mentioned classification techniques are based on color edge detection, which is, in general, dependent on the appropriate setting of threshold values to determine the edge maps. This threshold is found, in general, by trial-and-error. For general video segmentation and content recognition, manual settings of thresholds should be avoided. Therefore, an automatic way for threshold value selection is required.

In this paper, we aim at automatically classifying the physical nature of edges in images using color and reflectance information. To achieve this, we first propose a novel framework to compute edges by automatic gradient thresholding. Then, we present a taxonomy on edge types based upon the sensitivity of edges with respect to different imaging variables. Finally, a parameter-free edge classifier is provided labeling color transitions into one of the following types: (1) shadow-geometry edges, (2) highlight edges, (3) material edges. The proposed method is empirically verified on video sequences recorded from complex real world scenes.

The paper is organized as follows. In section 2, the basics on reflection are discussed first. Further, different color models are presented and a taxonomy on color invariance is given. In section 3, computational methods are proposed to get to color invariant gradients. Next, in section 4, error estimation and propagation is discussed. In section 5, the color edge classification scheme is proposed. Finally, in section 6, experiments are conducted.

\section{Photometric Invariance}

In Gevers and Smeulders [6] different color models are proposed which show some degree of invariance for the purpose of object recognition. In this paper, we use the different color models for the purpose of color edge classification in video sequences. Therefore, in this section, we first 
reconsider the basic reflection definitions in section 2.1 . Then, we discuss the different color models in section 2.2 and their sensitivity with respect to the imaging conditions. We conclude this section with a taxonomy on photometric invariance.

\subsection{Basic Reflection Definitions}

The reflection from inhomogeneous dielectric materials under white or spectrally smooth illumination is given by [7]

$$
\omega_{k}=G_{B}(\vec{n}, \vec{s}) E \int_{\lambda} B(\lambda) F_{k}(\lambda) d \lambda+G_{S}(\vec{n}, \vec{s}, \vec{v}) E S F
$$

for $\omega_{k} \in\{R, G, B\}$ giving the red, green and blue sensor response of an infinitesimal matte surface patch under the assumption of a white or spectrally smooth light source. Spectral sensitivities are given by $f_{R}(\lambda), f_{G}(\lambda)$ and $f_{B}(\lambda)$ respectively, where $\lambda$ denotes the wavelength. $B(\lambda)$ is the surface albedo. Further, $E$ denotes the white light source and $S$ is the Fresnel reflectance. These are constant over the wavelengths assuming white or spectrally smooth illumination (i.e. approximately equal/smooth energy density for all wavelengths within the visible spectrum) and the neutral interface reflection (NIR) model (i.e. $S(\lambda)$ has a constant value independent of the wavelength). Consequently, we have $E(\lambda)=E$ and $S(\lambda)=S$. Further, $\vec{n}$ is the surface patch normal, $\vec{s}$ is the direction of the illumination source, and $\vec{v}$ is the direction of the viewer. Finally, geometric terms $G_{B}$ and $G_{S}$ denote the geometric dependencies on the body and surface reflection component.

\subsection{Color Models}

We focus on normalized color $c_{1} c_{2}$ defined by [6]

$$
\begin{aligned}
& c_{1}(R, G, B)=\arctan \left(\frac{R}{B}\right) \\
& c_{2}(R, G, B)=\arctan \left(\frac{G}{B}\right) .
\end{aligned}
$$

Further, we consider the two-dimensional opponent color space (leaving out the intensity component here) defined by

$$
\begin{gathered}
o_{1}(R, G, B)=(R-G) / 2 \\
o_{2}(R, G, B)=B / 2-(R+G) / 4 .
\end{gathered}
$$

The opponent color space is well-known and has its fundamentals in human perception.

Consider the body reflection term of eq. (1)

$$
\beta_{k}(\vec{x})=G_{B}\left(\vec{x}, \vec{n}, \overrightarrow{)} E(\vec{x}) \int_{\lambda} B(\vec{x}, \lambda) F_{k}(\lambda) d \lambda\right.
$$

giving the $k t h$ sensor response of an infinitesimal matte surface patch under the assumption of a white light source. Again, for a color camera we have $k=\{R, G, B\}$.

The body reflection component describes the way light interacts with a dull surface. The light spectrum $E$ falls on a surface $B$. The geometric and photometric properties of the body reflection depends on many factors. If we assume a random distribution of the pigments, the light exits in random directions from the body. In this simple case, the distribution of exiting light can be described by Lambert's law. Lambertian reflection models dull, matte surfaces which appear equally bright regardless from angle they are viewed. They reflect light with equal intensity in all directions. As a consequence, a uniformly colored surface which is curved (i.e. varying surface orientation) gives rise to a broad variance of $R G B$ values. The same argument holds for intensity $I$.

In contrast, the $c_{1} c_{2}$ color model is a photometric invariant for matte, dull objects cf. eq. ( 6) and eqs. (2) and (3)

$$
\begin{gathered}
c_{1}\left(\beta_{R}, \beta_{G}, \beta_{B}\right)= \\
\arctan \left(\frac{G_{B}\left(\vec{n}, \overrightarrow{\$} E K_{R}(\lambda)\right.}{G_{B}\left(\vec{n}, \overrightarrow{\$} E K_{B}(\lambda)\right.}\right)=\arctan \left(\frac{K_{R}(\lambda)}{K_{B}(\lambda)}\right) \\
c_{2}\left(\beta_{R}, \beta_{G}, \beta_{B}\right)= \\
\arctan \left(\frac{G_{B}\left(\vec{n}, \overrightarrow{\$} E K_{G}(\lambda)\right.}{G_{B}\left(\vec{n}, \overrightarrow{\$} E K_{B}(\lambda)\right.}\right)=\arctan \left(\frac{K_{G}(\lambda)}{K_{B}(\lambda)}\right)
\end{gathered}
$$

where

$$
K_{C}(\lambda)=\int_{\lambda} B(\lambda) F_{C}(\lambda) d \lambda \text { for } C \in\{R, G, B\}
$$

is the compact formulation depending on the sensors and surface albedo only. Note that the dependency on illumination, object pose, camera position, and object shape is factored out i.e. $c_{1} c_{2}$ is only dependent on the sensors and the surface albedo.

For shiny surfaces, $\mathrm{O}_{1} \mathrm{O}_{2}$ is independent of highlights as follows from substituting eq. (1) in eqs. (4) and (5)

$$
\begin{gathered}
o_{1}\left(\omega_{R}, \omega_{G}, \omega_{B}\right)= \\
\left(\left(G_{B}(\vec{n}, \vec{s}) E \int_{\lambda} B(\lambda) F_{R}(\lambda) d \lambda+G_{S}(\vec{n}, \vec{s}, \vec{v}) E S F\right)-\right. \\
\left.\left(G_{B}(\vec{n}, \vec{s}) E \int_{\lambda} B(\lambda) F_{G}(\lambda) d \lambda+G_{S}(\vec{n}, \vec{s}, \vec{v}) E S F\right)\right) / 2= \\
\left(G_{B}(\vec{n}, \vec{s}) E \int_{\lambda} B(\lambda) F_{R}(\lambda) d \lambda-G_{B}(\vec{n}, \vec{s}) E \int_{\lambda} B(\lambda) F_{G}(\lambda) d \lambda\right) / 2
\end{gathered}
$$

Equal argument also holds for $o_{2}$. Note that $o_{1} O_{2}$ is still dependent on $G_{B}(\vec{n}, \vec{\xi})$ and $E$, and consequently being sensitive to object geometry and shading.

In conclusion, $c_{1} c_{2}$ varies with a change in material and highlights, $O_{1} O_{2}$ with a change in material and objectgeometry, and $R G B$ varies with a change in material, highlights and object-geometry. 


\section{Photometric Invariant Gradients}

A number of well established techniques for edge detection in ordinary (one-band) images is available, [1], [2]. However, for color edge detection, the computation of the gradient magnitude is more complex due to the multi-valued nature of a color image. In this paper, we compute the distance in color space by the Euclidean metric over the various channels.

Further, the color channels of an image are differentiated in the $x$ and $y$ direction by applying the Canny's edge detector (derivative of the Gaussian) with non-maximum suppression in a standard way to obtain the gradient magnitudes at local edge maxima denoted by $\left(\frac{\partial c_{i}}{\partial x}, \frac{\partial c_{i}}{\partial y}\right)$. Here, $c_{i}$ is the notation for a particular color channel.

Then, the modulus of the gradient $\nabla F$ of the color planes is obtained by taking the Euclidean distance

$$
\nabla F=\sqrt{\sum_{i=1}^{N}\left[\left(\frac{\partial c_{i}}{\partial x}\right)^{2}+\left(\frac{\partial c_{i}}{\partial y}\right)^{2}\right]}
$$

where $N$ is the dimensionality of the color space.

Often false edges are introduced due to sensor noise. These false edges are usually eliminated by using a threshold value determining the minimum acceptable gradient modulus. In this paper, we aim at providing a computational framework to determine automatically this local threshold value. To achieve this, sensor noise characteristics on color transformation are studied in the next section.

\section{Error Propagation}

In this paper, we assume that the noise is normally distributed, because the most frequently occurring noise is additive Gaussian noise. It is widely used to model thermal noise and is the limiting behavior of photon counting noise and film grain noise.

Then, let the result of a number of measurements of a random quantity $u$ be given by

$$
\hat{u}=u_{\text {best }} \pm \sigma_{u}
$$

where $u_{\text {best }}$ is the average value which is the best estimate for the quantity $u$ and $\sigma_{u}$ the standard deviation denoting the uncertainty or error in the measurement of $u$. Suppose that $u, \cdots, w$ are measured with corresponding uncertainties $\sigma_{u}, \cdots, \sigma_{w}$, and that the measured values are used to compute the function $q(u, \cdots, w)$. If the uncertainties in $u, \cdots, w$ are independent, random and relatively small, then the standard deviation or the so-called predicted uncertainty in $\hat{q}$ is given by [8]

$$
\sigma_{q}=\sqrt{\left(\frac{\partial q}{\partial u} \sigma_{u}\right)^{2}+\cdots+\left(\frac{\partial q}{\partial w} \sigma_{w}\right)^{2}}
$$

where $\partial q / \partial u$ and $\partial q / \partial w$ are the partial derivatives of $q$ with respect to $u$ and $w$. In any case, the uncertainty in $q$ is never larger than the ordinary sum

$$
\sigma_{q} \leq\left|\frac{\partial q}{\partial u}\right| \sigma_{u}+\cdots\left|\frac{\partial q}{\partial w}\right| \sigma_{w},
$$

if and only if the uncertainties $\sigma_{u}, \cdots, \sigma_{w}$ are relatively small. Assuming normally distributed random quantities, a way to calculate the standard deviations $\sigma_{R}, \sigma_{G}$, and $\sigma_{B}$ is to compute the mean and variance estimates derived from a homogeneously colored surface patch in an image under controlled imaging conditions. Although eqs. (13) and (14) are deduced for random errors, they have been used as universal formulas for all kinds of errors.

After calculating the noise variance, the uncertainty of $c_{1} c_{2}$ can be found by substitution of (2) and (3) into (13) as

$$
\begin{gathered}
\sigma_{c_{1}}=\sqrt{\frac{R^{2} \sigma_{B}^{2}+B \sigma_{R}^{2}}{\left(R^{2}+B^{2}\right)^{2}}} \\
\sigma_{c_{2}}=\sqrt{\frac{G^{2} \sigma_{B}^{2}+B \sigma_{G}^{2}}{\left(G^{2}+B^{2}\right)^{2}}}
\end{gathered}
$$

where $\sigma_{R}^{2}, \sigma_{G}^{2}$ and $\sigma_{B}^{2}$ denote the sensor noise variance, and $\sigma_{c_{1}}$ and $\sigma_{c_{2}}$ represent the uncertainty (standard deviation) in the normalized red and green color components, respectively. From the analytical study of eqs. (15) and (16), it can be derived that normalized color becomes unstable around the black point $R=G=B=0$.

Further, the uncertainty of the $o_{1} O_{2}$ opponent coordinates is given as

$$
\begin{gathered}
\sigma_{o_{1}}=\frac{1}{2} \sqrt{\sigma_{G}^{2}+\sigma_{R}^{2}} \\
\sigma_{o_{2}}=\frac{1}{2} \sqrt{4 \sigma_{B}^{2}+\sigma_{G}^{2}+\sigma_{R}^{2}}
\end{gathered}
$$

which is relatively stable at all $R G B$ points.

Further, to propagate the uncertainties from these color components through the Gaussian gradient modulus, the uncertainty in the gradient modulus is determined by convolving the confidence map with the Gaussian coefficients. This results from the uncertainty in sums and differences as follows [8]. If several quantities

$$
\hat{u}_{1}, \cdots, \hat{u}_{N}
$$

are measured with uncertainties

$$
\sigma_{\hat{u}_{1}}, \cdots, \sigma_{\hat{u}_{N}}
$$

to compute

$$
q=\hat{u}_{1}+\hat{u}_{2} \cdots+\left(\hat{u}_{N-1}+\hat{u}_{N}\right)
$$


then the uncertainty in the computed value of $q$ is the sum

$$
\sigma_{q}=\sigma_{\hat{u}_{1}}+\sigma_{\hat{u}_{2} \ldots}+\sigma_{\hat{u}_{N-1}}+\sigma_{\hat{u}_{N}}
$$

As a consequence, we obtain:

$$
\sigma_{\nabla F} \leq \frac{\sum_{i}\left[\left(\partial c_{i} / \partial x\right) \cdot \sigma_{\partial c_{i} / \partial x}+\left(\partial c_{i} / \partial y\right) \cdot \sigma_{\partial c_{i} / \partial y}\right]}{\sqrt{\sum_{i}\left[\left(\partial c_{i} / \partial x\right)+\left(\partial c_{i} / \partial\right)\right]}}
$$

where $i$ is the dimensionality of the color space and $c_{i}$ is the notation for particular color channels. In this way, the effect of measurement uncertainty due to noise is propagated through the color gradients.

\section{Automatic Edge Thresholding and Classification}

In this section, techniques are presented to automatically select the gradient threshold value. The threshold value is computed locally in an adaptive way. In fact, the amount of uncertainty at image locations will steer the threshold value. Finally, a color edge taxonomy is presented on which the novel rule-based edge classifier is based on.

\subsection{Parameter-Free Edge Thresholding}

From (23) the uncertainty associated with the gradient modulus is known. Color edges are thresholded taking this uncertainty into account. As we assume that the noise is normally distributed (Gauss distribution), it is well-known that $99 \%$ of the values fall within a $3 \sigma$ margin. If a gradient modulus is detected which exceeds $3 \sigma_{\nabla F}$, then there is a chance of $1 \%$ that this gradient modulus corresponds to no color transition:

$$
\nabla C(x, y)= \begin{cases}1 & \text { if } \nabla F(x, y)>3 \sigma_{\nabla F}(x, y) \\ 0 & \text { otherwise }\end{cases}
$$

deriving a local threshold value.

The novelty of our approach is that the threshold value is automatically and locally adapted to the amount of uncertainty of the color invariant edge. For example, for $c_{1} c_{2}$ edges (unstable near the black point) at pixels with low intensity, the threshold value is automatically augmented. In this way, a local, noise-adaptive and automatic thresholding scheme is obtained.

\subsection{Reflectance Based Edge Classification}

In the previous sections, the effect of varying imaging circumstances have been analyzed first: $c_{1} c_{2}$ varies with a change in material and highlights, $o_{1} O_{2}$ with a change in material and object geometry, and $R G B$ varies with a change in material, highlights and object geometry. Further, color invariant edges have been computed with their associated uncertainty.

As a consequence, we conclude that $\nabla \mathcal{C}_{R G B}$ (denoting the edge map in $R G B$-space with noise-adaptive thresholding corresponding to eq. (24)) measures the presence of (1) shadow or geometry edges, (2) highlight edges, (3) material edges. Further, $\nabla \mathcal{C}_{c_{1} c_{2}}$ (denoting the edge map in $c_{1} c_{2}$ normalized space) measures the presence of (2) highlight edges, (3) material edges. Finally, $\nabla \mathcal{C}_{o_{1} o_{2}}$ measures the presence of (1) shadow or geometry edges, (3) material edges.

In this way, a taxonomy of color edge types is obtained, see figure 1. The color edge taxonomy is based upon the sensitivity of the color gradients with respect to the following imaging conditions: object geometry, shadows, highlights, and material.

\begin{tabular}{|c|c|c|c|c|}
\hline & shape edges & shadow edges & highlight edges & material edges \\
\hline$\nabla \mathrm{C}_{\mathrm{RGB}}$ & + & + & + & + \\
\hline$\nabla \mathrm{C}_{\mathrm{c} 1 \mathrm{c} 2}$ & - & - & + & + \\
\hline$\nabla \mathrm{C}_{\mathrm{O} 1 \mathrm{o} 2}$ & + & + & - & + \\
\hline
\end{tabular}

Figure 1: Taxonomy of color edges based upon the sensitivity of the different color edge models with respect to the imaging conditions. - denotes invariant and + denotes sensitivity of the color edge model to the imaging condition.

Then the rule-based reflectance classifier is as follows:

IF $\nabla \mathcal{C}_{R G B} \neq 0$ AND $\nabla \mathcal{C}_{c_{1} c_{2}}=0$

$T H E N$ classify as shadow or geometry edge

ELSE

IF $\nabla \mathcal{C}_{c_{1} c_{2}} \neq 0$ AND $\nabla \mathcal{C}_{o_{1} o_{2}}=0$

THEN classify as highlight edge

ELSE

classify as material edge

only computed at color edge maxima using non-maxima suppression. Note again that the color edges are computed by eq. (24). In this way, color edges and threshold values are automatically calculated in a parameter-free setting.

\section{Experiments}

In this paper, experiments are conducted on still images (for illustration purposes) and video sequences recorded from complex scenes. To this end, in section 6.1, we focus on color images taken from simple objects. In section 6.2, video sequences are taken into account. 


\subsection{Automatic Edge Classification in Still Im- ages}

For this experiment, an image is taken of two homogeneously colored plastic toys (red and green) against a blue paper background. The experiments are performed using a Sony 3CCD color camera XC-003P, Matrox Corona Framegrabber, and four Osram 18 Watt "Lumilux deLuxe daylight" fluorescent light sources.

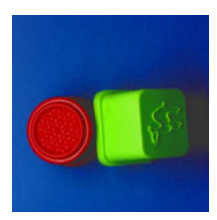

(a) Image

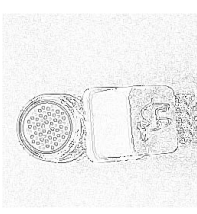

(b) $\nabla_{c_{1} c_{2}}$

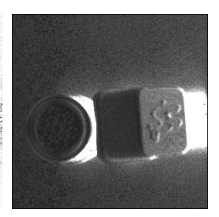

(c) $\sigma_{\nabla_{c_{1} c_{2}}}$

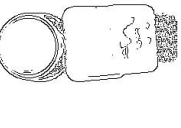

(d) Thres.
Figure 2: Edges in normalized (b) color space, and the associated uncertainty $(c)$. In $(d)$, the result of manual global thresholding.

The image is shown in figure 2.a. The red object shows two circles enclosing a number of small specularities. A homogeneous, almost black, shadow region is visible at the right side of the green toy. The edge map, computed in the normalized color space, is shown in figure 2.b. As expected, the normalized color is sensitive to highlights. This results in the specularities at the red object. Further, it is experimentally established that the normalized color space is highly unstable at dark colors (i.e. low intensity). The uncertainty map of $c_{1} c_{2}$ edges is shown in figure 2.c. Note that uncertain values are depicted in black. In this way, regions with high intensity, in the original image, correspond to dark regions in the uncertainty map $\sigma_{\nabla_{c_{1} c_{2}}}$. In figure 2.d, the edge map is shown by thresholding the normalized color gradient by using a global threshold value. The most optimal threshold value has been selected by visual inspection. As color invariant instabilities are not stationary over the image (i.e. at each image location a different threshold is required), the specularities on the red cup having gradient moduli below the threshold value are shown up, whereas the noise edges in the dark region have gradient moduli values exceeding the threshold value. The experiment shows the inappropriateness of the use of a global (manual) threshold due to the local instabilities of color invariant edges.

The result of the newly proposed noise-adaptive thresholding scheme is shown in figure 3. In figure figure 3.a the gradient is shown computed for the $R G B$ color space. The image shows that many false edges are correctly suppressed while edges caused by material, geometry and specularity transitions are retained. Figure 3.b shows the result

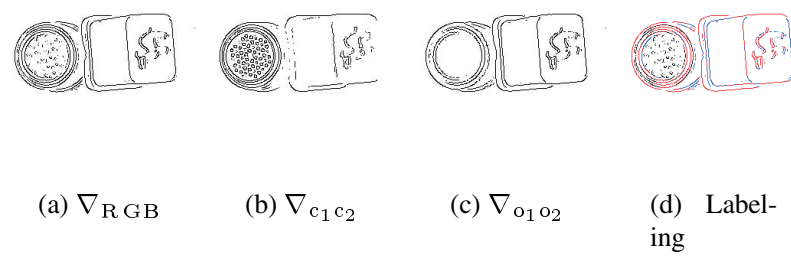

Figure 3: Results of automatic local thresholding in sensor (a), normalized (b) and opponent (c) color space. Figure (d) shows the result of color edge classification. Here, black edges are highlight edges, blue edges are geometry or shadow edges, red edges are material transitions.

for normalized color space. Here, noise-adaptive thresholding correctly discards the edges present in the edge map of figure 2.b while retaining the highlight edges on the bottom of the red cup. Figure 3.c shows the result of automatic thresholding for the opponent color space. As expected, the color space is invariant for highlights, which consequently do not show up in the edge map. Note that the opponent color space still depends on shadows and object geometry. Finally, in figure 3.d, the result of automatic edge labeling is shown. Black edges correspond to highlight edges, red edges to material transitions, and blue edges to geometry changes. It is shown that the proposed method successfully classifies color edges in still images without the need for parameter settings.

\subsection{Automatic Edge Classification in Video}

In figure 4.a, six frames are shown from a standard video often used as a test sequence in the literature. It shows a person against a textured background playing ping-pong. The size of the image is $260 \times 135$. The images are of low quality. The frames are clearly contaminated by shadows, shading and inter-reflections. Note that each individual object-parts (i.e. T-shirt, wall and table) is painted homogeneously with a distinct color. Further, that the wall is highly textured. The results of the proposed reflectance based edge classifier are shown in figure 4.b. As no highlights are present in the scene, the edge classifier discriminates edges in the color image to be one of the following types: (1) material edges shown in figure 4.c, and (2) shadow or geometry edges shown in figure 4.d. As one can see, the cast shadow of the person on the wall is well detected and classified. Also the geometry transitions on the T-shirt have been classified successfully. Material edges, as shown in figure 4.c, are well-defined ignoring radiometrical effects. Only inter-reflections and smoothly changing shading dis- 


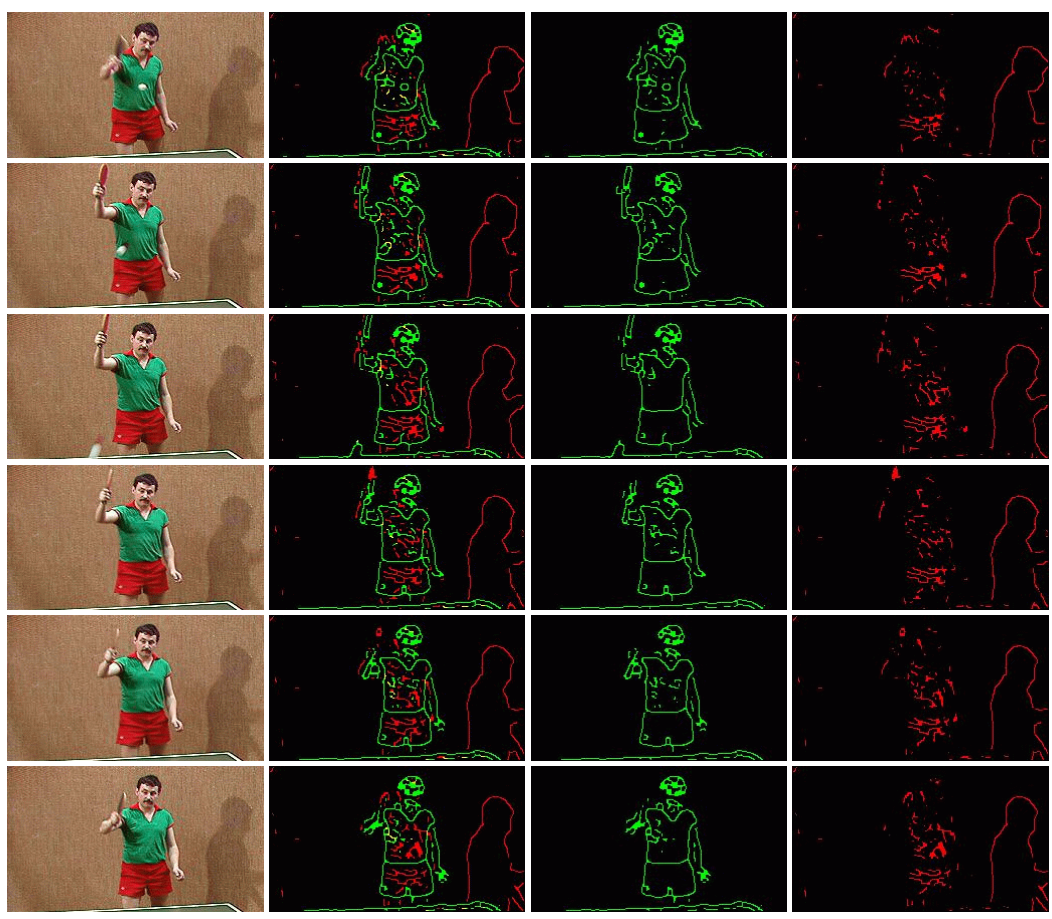

Figure 4: Frames from a video showing a person against a textured background playing ping-pong. From left to right column. a. Original color frame. $b$. Classified edges. c. Material edges. $d$. Shadow and geometry edges.

turb the edge map slightly. From the observed results, it is concluded that the edge classifier discriminates the various edge types satisfactory. Only minor errors are caused when intensity change smoothly over a wide image range due to the local behavior of the edge classifier.

\section{Conclusion}

Color information has been used to classify the physical nature of a color edge. A novel framework has been proposed for color edge detection and automatic noise-adaptive thresholding. The framework is derived from sensor noise analysis and propagation. Further, a parameter-free color edge classifier has been proposed labeling color transitions into the following types: (1) shadow, geometry or shading edges, (2) highlight edges, (3) material edges. From the theoretical and experimental results it is concluded that proposed method successfully classifies color edges in video without the need for parameter settings.

\section{References}

[1] J. Canny, A Computational Approach to Edge Detection, IEEE Transactions on Pattern Analysis and Machine Intelligence, Vol. 8, No. 6, pp. 679-698, 1986.
[2] D. Ziou and S. Tabbone, Edge Detection Techniques An Overview, Pattern Recognition and Image Analysis, 8(4), pp. 537-559, 1998.

[3] W. H. Tsang and P. W. M. Tsang, Suppression of False Edge Detection due to Specular Reflection in Color Images, Pattern Recognition Letters, 18:165171, 1997.

[4] W. Zhang and F. Bergholm, Multi-scale Blur Estimation and Edge Type Classification for Scene Analysis, International Journal of Computer Vision, 24(3), pp. 219-250, 1997.

[5] J. Stander, R. Mech, J. Ostermann, Moving Cast Shadows Detection for Object Segmentation, IEEE Transactions on Multimedia, pp. 65-76, 1999.

[6] Th. Gevers and A.W.M.Smeulders, Color Based Object Recognition, Pattern Recognition, vol. 32, pp. 453-464, 1999.

[7] S.A. Shafer, Using Color to Separate Reflection Components, Color Research and Applications, 10(4), pp. 210-218, 1985.

[8] J. R. Taylor. An Introduction to Error Analysis, University Science Books, Mill Valley, CA, 1982. 